\title{
The Excess Radio Background and Fast Radio Transients
}

\author{
John Kehayias, ${ }^{*}$ Thomas W. Kephart, ${ }^{\dagger}$ and Thomas J. Weiler ${ }^{\ddagger}$ \\ Department of Physics and Astronomy, Vanderbilt University \\ Nashville, TN 37235, United States
}

(Dated: October 2, 2018)

\begin{abstract}
In the last few years ARCADE 2, combined with older experiments, has detected an additional radio background, measured as a temperature and ranging in frequency from $22 \mathrm{MHz}$ to $10 \mathrm{GHz}$, not accounted for by known radio sources and the cosmic microwave background. One type of source which has not been considered in the radio background is that of fast transients (those with event times much less than the observing time). We present a simple estimate, and a more detailed calculation, for the contribution of radio transients to the diffuse background. As a timely example, we estimate the contribution from the recently-discovered fast radio bursts (FRBs). Although their contribution is likely 6 or 7 orders of magnitude too small (though there are large uncertainties in FRB parameters) to account for the ARCADE 2 excess, our development is general and so can be applied to any fast transient sources, discovered or yet to be discovered. We estimate parameter values necessary for transient sources to noticeably contribute to the radio background.
\end{abstract}

\section{INTRODUCTION}

Radio astronomy and astrophysics have an influential history, intertwined with astronomy, cosmology, particle, and nuclear physics. Today there are mysterious new sources being discovered in the radio universe. In this work we will focus on two recent and exciting developments, namely an observed and unexplained excess in the radio background (ERB) and the discovery of fast radio bursts (FRBs). We use the latter fast radio transient (FRT) sources as the example for our modeling.

The ARCADE 2 (Absolute Radiometer for Cosmology, Astrophysics and Diffuse Emission) experiment reported [1] an excess in temperature, after subtracting the cosmic microwave background (CMB) and accounting for other known backgrounds, in measurements at frequencies from $\sim 3-10 \mathrm{GHz}$. When combined with previous data and analysis in the literature there is an excess that extends from $22 \mathrm{MHz}$ to nearly $10 \mathrm{GHz}$. This is well fit by a power law in absolute temperature versus frequency with the following form [1]

$$
T=(24.1 \pm 2.1 \mathrm{~K})\left(\frac{\nu}{310 \mathrm{MHz}}\right)^{-2.5990 \pm 0.0366} .
$$

While ARCADE 2 measures a temperature for the radio background, transient sources like FRBs are measured by other experiments in units of spectral irradiance or spectral flux density: Janskys, ${ }^{1}$ where $1 \mathrm{Jy} \equiv$

\footnotetext{
* john.kehayias@vanderbilt.edu

† thomas.w.kephart@vanderbilt.edu

¥ tom.weiler@vanderbilt.edu

${ }^{1}$ For our fellow particle physicists, one might be tempted to identify the energy implicit in Watts with that in the Hertz in the denominator, but these energies do not "cancel." One should think of the Watts as the power that is received by the radio telescope at a particular frequency in Hertz (and per unit area). The bandwidth (frequency range of observation), measured in Hertz as well as the observing area, will directly affect the power
}

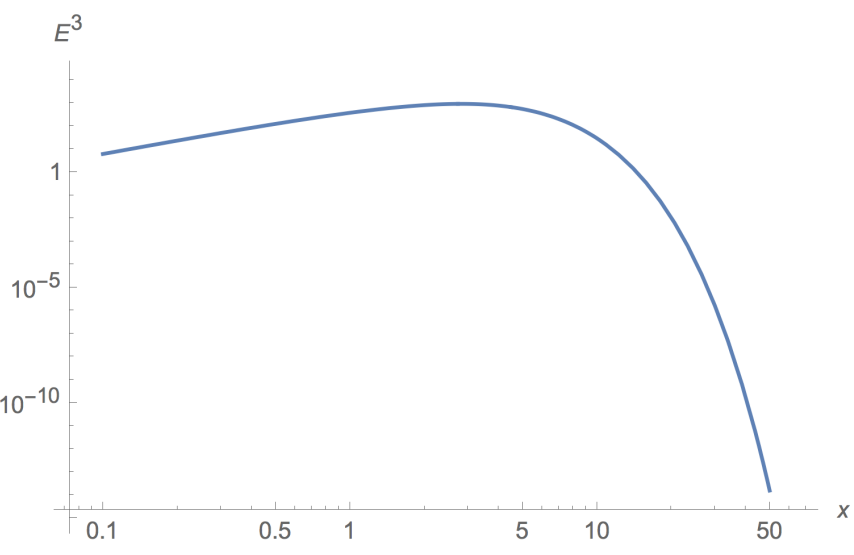

FIG. 1. A log-log plot of eq. (2) versus $x$, with $T=10$.

$10^{-26} \mathrm{~W} \mathrm{~m}^{-2} \mathrm{~Hz}^{-1}$. To convert these temperature measurements into a flux density, we show the standard distribution function (i.e. Planck radiation law) for a massless Bose particle (here a photon) as a function of the dimensionless variable $x \equiv h \nu / k_{B} T$ in Fig. 1 . The energy density per frequency distribution, $\mathrm{d} \rho / \mathrm{d} \nu$, is given by

$$
\frac{\mathrm{d} \rho}{\mathrm{d} \nu}=\frac{2}{\pi} \frac{T^{3} x^{3}}{e^{x}-1}=\frac{2 h \nu^{3}}{c^{2}} \frac{1}{e^{\frac{h \nu}{k_{B} T}}-1},
$$

with the first form written in natural units $(\hbar=c=$ $k_{B}=1$ ) with overall units of energy ${ }^{3}$, and the second is the typical textbook form with the constants restored and units proportional to Janskys. Note that when $T$ is allowed to vary with frequency, as in the ERB data, then the spectral form will differ from the fixed distribution shown in Figure 1.

received, and thus it is more sensible to express the radio unit as power per unit frequency and area (an energy flux density): the Jansky. 
This excess background has persisted and has been difficult to understand despite different analyses (see, e.g., $[2,3]$ ), and remains of unknown origin (for a recent study see, e.g., [4] and references therein). If the background comes from additional extragalactic sources, they must be very numerous and faint, e.g. [2, 5]. Additionally, the background appears to be extremely smooth, which puts further constraints on the size and cosmological distribution of such background sources [6]. There have been several different proposals for an alternative origin for this radio background, including dark matter [7], but the question is still open.

More recently there has been the discovery, first with the Parkes telescope [8-10] and then at Arecibo [11], of a new class of radio objects, dubbed "fast radio bursts" (FRBs) due to their only known activity occurring on the millisecond Earth-time scale (FRB discoveries have continued as well [12]). Based upon their dispersion measures (DMs), ${ }^{2}$ these FRBs are believed to be extragalactic in origin (although this has been challenged, see, e.g., [13]). The physical process powering an FRB is so far unknown, with proposals ranging from neturon stars [14], axions [15], and black holes [9, 16] to several others [17]. It is further inferred that these unknown objects release $\sim 10^{38}-10^{40}$ erg during their millisecond duration, corresponding to a typical peak flux density of $>0.4 \mathrm{Jy}$. While there are only a few observed FRBs (11 at this moment; an up to date list with measured parameters and references is available at http://astro.phys.wvu.edu/FRBs/), a rough estimate of the rate for these events gives of order 10,000 per day in the whole sky out to $z=0.75$ (recent studies, such as [18], put the rate a bit lower, though still consistent with earlier results and of negligible effect for our present analysis).

Motivated by FRBs, we will consider general fast transient radio sources as contributing to the ERB. By "fast" we mean that the timescale of such an event is much less than the time of observations (which is of order an hour for ARCADE 2). Such sources have not been considered in the past for calculating the diffuse radio background; we will adjust the steady state radio source calculation to accommodate the transient contribution.

This paper is organized as follows. First, in Section II A, we will introduce the basic calculation for radio source counts for the radio background, and modify it to account for fast transients. While the FRB contribution to the excess radio background is several orders of magnitude too small to make detectable contribution, a

\footnotetext{
${ }^{2}$ Without candidate source galaxies for FRBs thus far, the only way to estimate their distance is through their dispersion measure. The DM is determined by the electron density along the line of sight, which delays lower frequency signals compared to higher frequencies. Given an estimate for the electron contribution from the galaxy, the large DMs of FRBs lead to the inference of extragalactic sources.
}

more detailed calculation for transient sources, e.g. similar to the calculation for supernova neutrinos, may be useful. In Section II B we present a more complete analysis. We then compare a general fast source to the excess background to derive approximate parameters for such a source to contribute to the excess at the percent level or more in Section III. Finally, in Sections IV and V we discuss our results and future directions before concluding.

\section{A RADIO BACKGROUND FROM FAST TRANSIENTS}

Fast transient sources have not been considered as a contribution to the observed ERB. One might ask if they can contribute appreciably to the radio background. In this Section we will first use a simplified analysis to estimate the contribution from fast transients before providing a more comprehensive calculation. Fast transient sources need to be treated slightly differently in the usual radio background calculation in order to properly account for their event rate and individual event time. For a recent review of FRT searches and related bounds, see [19] and references therein.

\section{A. An Estimate}

For a typical source (static, or slowly changing, compared to typical observation times), the contribution to the cosmic radio background (CRB) (see e.g. [2]) is

$$
B_{\mathrm{CRB}}=\int_{S_{\min }}^{\infty} S \frac{\mathrm{d} N}{\mathrm{~d} S} \mathrm{~d} S,
$$

where $S$ is the flux density at Earth (energy/(time-area-frequency), e.g. in units of Janskys), and $\frac{\mathrm{d} N}{\mathrm{~d} S}$ is the differential number of sources at that flux density. The unit for radio observations, the Jansky, implicitly has a time such that multiplying by an observation time, area, and frequency bandwidth, yields the total energy observed.

For transient sources, however, there are now two timescales which are introduced: the duration of the source emission and the inverse rate of sources appearing in the sky. For fast sources (where "fast" means short compared to the observation time, e.g. ARCADE 2 here), the entire energy of each event is observed. Instead of a flux density per source, we should use an energy density: we multiply $S$ by $\delta t$, the time of the source mission. We still want the same units at the end, so we need an inverse time, which comes from the number per flux density being replaced by a rate per flux density (i.e. a number per energy density), $\mathrm{d} N / \mathrm{d} S \mathrm{~d} t$, with units of per second after integration over $S$. In other words, the source flux density is to be multiplied by the duration of the transient source, while the number density becomes a rate. 
We can write this as

$$
B_{\mathrm{CRB}}^{\mathrm{FRT}}=\int_{S_{\min }}^{\infty} S \delta t \frac{\mathrm{d}^{2} N}{\mathrm{~d} S \mathrm{~d} t} \mathrm{~d} S,
$$

The result can properly be interpreted as the incoming energy flux density, just as in Eq. (3). ${ }^{3}$

Let us consider observations at one particular frequency, $\sim 1.4 \mathrm{GHz}$, for which there is observation of both the $\mathrm{ERB}^{4}$ and FRBs. The radio background can be expressed as $10^{4} \mathrm{Jy} / \mathrm{sr}$ (e.g., see [2]). The estimated event rate of FRBs of $10^{4}$ per day in the whole sky (for $S>1 \mathrm{Jy}$ ) corresponds to $10^{-2} \mathrm{~s}^{-1} \mathrm{sr}^{-1}$ in units more useful for us. As a lower estimate on the FRB contribution to the ERB at $1.4 \mathrm{GHz}$, we simply multiply this rate by the typical flux density times the millisecond duration, $1 \mathrm{Jy} \times 10^{-3} \mathrm{~s}$. Thus the FRB contribution has a lower bound of $\sim 10^{-9}$ times the observed radio background; this is a lower bound because the true contribution will be larger, from inclusion of further/dimmer events, as well as modeling the distribution of FRBs in redshift. (We will see in Section II B 1 that our more detailed calculation, using a model for the FRB spectrum and distribution, increases the FRB background by a factor of $\sim 100$.)

To verify this very rough estimate, we will assume a form for the differential number per flux density, and integrate backwards in time (outwards in space). (Since it is estimated from the DMs that the observed FRBs have red-shifts $\lesssim 1$, the red-shifting of energies, and dilation of time, are small.) With the assumptions that FRBs are standard candles observed at extragalactic distances (as is so far consistent with all available data), the nearby $(z<1$ or $S>1$ Jy) distribution approximately follows that of a static Euclidean universe, $\frac{\mathrm{d} N}{\mathrm{~d} S}=S^{-5 / 2}$. We normalize this distribution to the estimated FRB rate above, $\int_{1}^{\infty} \frac{\mathrm{d} N}{\mathrm{~d} S} \mathrm{~d} S=10^{-2} \mathrm{~s}^{-1} \mathrm{sr}^{-1}$. Inputting this assumption allows integration of eq. (3), and we find the FRB contribution matches our rough estimate of $10^{-9}$ of the observed radio excess.

\section{B. A More Detailed Calculation}

To calculate the radio background due to FRTs in the universe, we follow the same basic procedure as in, e.g., the neutrino background from supernovas (see [20] for a review). The two main ingredients are an event rate (number per volume per time) evolving in redshift, $R(z)$,

\footnotetext{
${ }^{3}$ Implicitly this is over the entire sky, but one can divide by $4 \pi$ or else use a rate which is per steradian.

${ }^{4}$ For now we will ignore any errors in the measurements, though the ERB data at $1.4 \mathrm{GHz}$ is consistent (within errors) with zero after additional source subtractions. One could use a different frequency for the ERB, with an assumption of the FRB spectrum, and would find similar results.
}

and the spectrum (energy per frequency) of an individual event, $F(\nu)$, with $\nu$ the frequency observed at Earth. The incoming isotropic flux background, in units of energy/(time $\cdot$ area $\cdot$ steradian $\cdot$ frequency), or $\mathrm{Jy} / \mathrm{sr}$, is then calculated by the following integral,

$$
B(\nu)=\frac{1}{4 \pi} \frac{c}{H_{0}} \int_{0}^{z_{\max }} \mathrm{d} z \frac{R(z) F\left(\nu^{\prime}\right)}{\sqrt{\Omega_{\mathrm{m}}(1+z)^{3}+\Omega_{\Lambda}}},
$$

where $c / H_{0}$ is the Hubble length, $z_{\max }$ is the redshift back to which we are integrating sources, $\nu^{\prime} \equiv \nu(1+z)$ is the blueshifted frequency, and $\Omega_{\mathrm{m}}$ and $\Omega_{\Lambda}$ are the energy densities of matter and dark energy, respectively.

To perform this calculation we will need to model our radio sources. First, let us consider how the sources are distributed in redshift. We will employ two models, one where the source distribution simply scales with the comoving volume as a standard candle (FRBs were modeled like this in [21]), and another which tracks the star formation rate. These models are shown in Figure 2.

In the first model we assume the sources have a constant number density in comoving volume, then the rate simply scales with the fraction of comoving volume out to $t_{f}(z)$. With

$$
D(z) \equiv c \int_{t_{0}}^{t_{f}} \frac{\mathrm{d} t}{a(t)}=\frac{c}{H_{0}} \int_{0}^{z} \mathrm{~d} z^{\prime} \frac{1}{\sqrt{\Omega_{\mathrm{m}}\left(1+z^{\prime}\right)^{3}+\Omega_{\Lambda}}}
$$

the comoving distance, the source rate is

$$
R_{\mathrm{CoV}}^{\mathrm{FRT}}(z)=N_{\mathrm{CoV}}^{\mathrm{FRT}} \frac{D(z)^{3}}{D\left(z_{*}\right)^{3}},
$$

where $N_{\mathrm{CoV}}^{\mathrm{FRT}}=R_{\mathrm{CoV}}^{\mathrm{FRT}}\left(z_{*}\right)$ is a normalization to be fixed by the source model or observation.

Alternatively, we model the source rate to be proportional to the star formation rate. There are several different parameterizations and we follow the one formulated in Table II of [22], but with some parameters slightly rounded as in [20],

$$
R_{\text {star }}(z) \propto \begin{cases}(1+z)^{3.28}, & z<1 \\ a_{1}(1+z)^{-0.26}, & 1<z<4.5 \\ a_{2}(1+z)^{-7.8}, & 4.5<z\end{cases}
$$

with $a_{1}=11.6$ and $a_{2}=4.45 \times 10^{6}$ fixed by continuity. The transient radio source rate is then

$$
R_{\mathrm{SF}}^{\mathrm{FRT}}(z)=N_{\mathrm{SF}}^{\mathrm{FRT}} R_{\mathrm{star}}(z),
$$

with a normalization denoted by $N_{\mathrm{SF}}^{\mathrm{FRT}}$.

Now we turn to modeling the spectral properties of the fast sources themselves. As the simplest model we will take the spectrum of the sources to follow a power law (in frequency) over some range of frequency. The free parameters will be the spectral index, overall normalization, and frequency range. Since we are studying the radio background over observations much longer than 
the source timescale, we will not need to directly assume this timescale. Instead, it is implicit in our normalization of the total energy. For simplicity we will assume the sources are either standard candles or consider all quantities to be typical or average values.

We write the spectrum as

$$
F(\nu)=F_{\text {norm }}\left(\frac{\nu}{\nu_{*}}\right)^{\alpha},
$$

with spectral index $\alpha$, reference frequency $\nu_{*}$, and normalization $E_{\text {norm }}$ with units of energy per unit frequency. The normalization is fixed by integrating the spectrum over its frequency range, $\nu_{\min }$ to $\nu_{\max }$, to determine the total energy released in an event,

$$
E_{\text {total }}=\int_{\nu_{\min }}^{\nu_{\max }} \mathrm{d} \nu F(\nu)
$$

\section{Fast Radio Burst Modeling}

Before considering a general (as yet unknown) source to fit to the excess radio background, we return to FRBs as an example of applying the above basic modeling. With the current data on FRBs, the consensus on the approximate rate of FRBs is about 10,000 per day in the whole sky, out to a redshift 0.75 . We will use this observed rate, expressed in more useful units as

$$
R_{\mathrm{FRB}}(z \leq 0.75) \equiv 2.40 \times 10^{-79} \mathrm{~s}^{-1} \mathrm{~m}^{-3},
$$

as the integrated rate to redshift 0.75 to fix the normalization.

If we assume FRBs are standard candles with a constant number density in comoving volume (as in [21]), their rate is modeled by eq. (7) with the normalization, $N_{\mathrm{CoV}}^{\mathrm{FRB}} \equiv 4.68 R_{\mathrm{FRB}}(z \leq 0.75)$ determined by setting $z_{*}=0.75$ in eq. (7) and integrating out to $z_{*}$. In our alternative model based on the star formation rate, eq. (8), the normalization, obtained by direct integration, is $N_{\mathrm{SF}}^{\mathrm{FRB}} \equiv 0.43 R_{\mathrm{FRB}}(z \leq 0.75)$.

The second set of parameters for FRBs is their typical energy output and spectrum. From the observed events thus far, the energy released is about $10^{31}$ to $10^{33} \mathrm{~J}$, or $10^{38}$ to $10^{40} \mathrm{erg}$. We use the FRB model in [21] as a reference point: a spectral index of -1.4 from $10 \mathrm{MHz}$ to $10 \mathrm{GHz}$, with a bolometric luminosity of $8 \times 10^{44} \mathrm{erg} / \mathrm{s}$, or a total energy of $8 \times 10^{34} \mathrm{~J}$ (using an event time of $1 \mathrm{~ms})$.

With these parameters we can calculate the FRB contribution to the radio background, again at $1.4 \mathrm{GHz}$. We find that the FRB contribution is approximately $3 \times 10^{-7}$ of the observed excess with a constant comoving volume event distribution, and $0.5 \times 10^{-7}$ when using an event rate proportional to the star formation rate.

Given the large uncertainties in what is known about FRBs, we can vary the parameters to some degree. From eq. (5) we know that any changes to the overall rate or

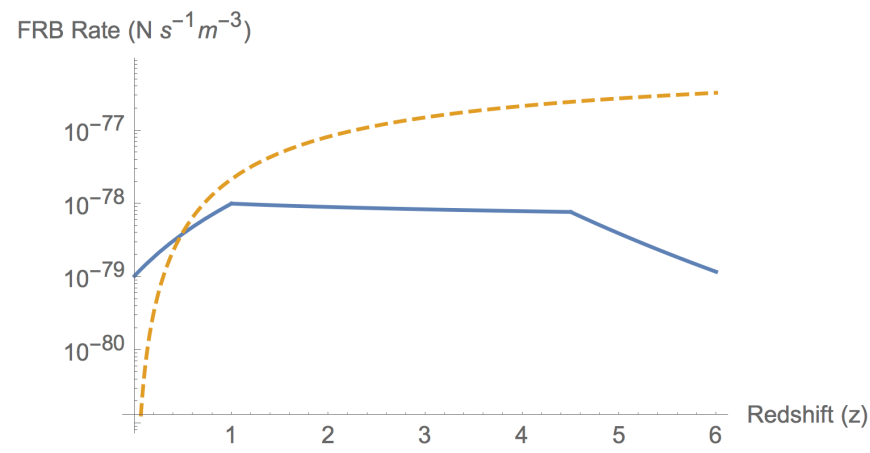

FIG. 2. A model for the FRB rate scaled with comoving volume (orange, dashed) and tracking the star formation rate (blue, solid). The rates are normalized such that their integral to $z=0.75$ matches the approximate observed rate.

energy of the radio transients will proportionally change the calculated background contribution. For instance, if the rate is underestimated by a factor of a few and the total energy is underestimated by an order of magnitude (both reasonable possibilities, given the observed values and error bars), the FRB background contribution could be $10^{-5}$ of the excess. While further tuning of parameters or modeling might improve this slightly, it seems unlikely that, without a drastic modification of what is known about FRBs, could they make up a significant proportion of the ERB.

\section{FITTING A FAST TRANSIENT SOURCE TO THE EXCESS BACKGROUND}

We will now fit the flux density of a new source of FRTs to the observed ERB from ARCADE 2 and previous experiments. These experiments report a temperature beyond the contribution from the CMB temperature of $2.725 \mathrm{~K}$ and the galactic foreground. We use "Fit 1 " of [23] to estimate the contribution from unresolved extragalactic discrete sources (see also [24] for resolving such sources). Using the Planck radiation law, Eq. (2), we convert these temperatures to an expected spectral flux density, in units of $\mathrm{Jy} / \mathrm{sr}^{5}$ The resulting data is shown in Table I. We note that the data point at $1.4 \mathrm{GHz}$ is consistent with zero given the errors on the measured temperature after the above subtractions. We do not include data at frequencies larger than $\sim 8 \mathrm{GHz}$ as they are also consistent with zero.

Fitting to these 8 data points, from $22 \mathrm{MHz}$ to $8 \mathrm{GHz}$, we find a good fit to the observed excess with the contribution from FRTs integrated back to redshift 6 .

First, we consider the case when we model the transient rate as the comoving volume. With the parameters

\footnotetext{
5 Note that the reported errors in temperature become asymmetric in radiance; we use the smaller of the $\mathrm{max} / \mathrm{min}$ errors for each data point as a common error for fitting purposes.
} 


\begin{tabular}{|c|c|c|c|}
\hline Frequency & Temperature $(\mathrm{K})$ & Final Temperature $(\mathrm{K})$ & Flux Density $(\mathrm{kJy} / \mathrm{sr})$ \\
\hline $22 \mathrm{MHz}$ & $20355 \pm 5181$ & 13479 & $200.430 \pm 77.043$ \\
$45 \mathrm{MHz}$ & $3864 \pm 502$ & 2866 & $178.300 \pm 31.200$ \\
$408 \mathrm{MHz}$ & $13.42 \pm 3.52$ & 8.107 & $41.410 \pm 18.000$ \\
$1.42 \mathrm{GHz}$ & $3.271 \pm 0.526$ & 0.4568 & $26.24 \pm 32.60$ \\
$3.20 \mathrm{GHz}$ & $2.787 \pm 0.010$ & 0.05205 & $2.667 \pm 1.380$ \\
$3.41 \mathrm{GHz}$ & $2.770 \pm 0.008$ & 0.03662 & $0.6777 \pm 0.4850$ \\
$7.98 \mathrm{GHz}$ & $2.761 \pm 0.013$ & 0.03516 & $(13.92 \pm 13.90) \times 10^{-3}$ \\
$8.33 \mathrm{GHz}$ & $2.743 \pm 0.015$ & 0.01725 & $(73.23 \pm 73.20) \times 10^{-6}$ \\
\hline
\end{tabular}

TABLE I. The ERB data used in this analysis. The data is from ARCADE 2 and older experiments (see Table 4 of Ref. [1] and references therein), with the first temperature being the reported temperature before subtractions, but accounting for the galactic foreground. The final temperature is after the CMB, and extragalactic sources have been subtracted (the error bars are the same as in the previous column). The flux density is derived from this final temperature and is the value used for fitting.

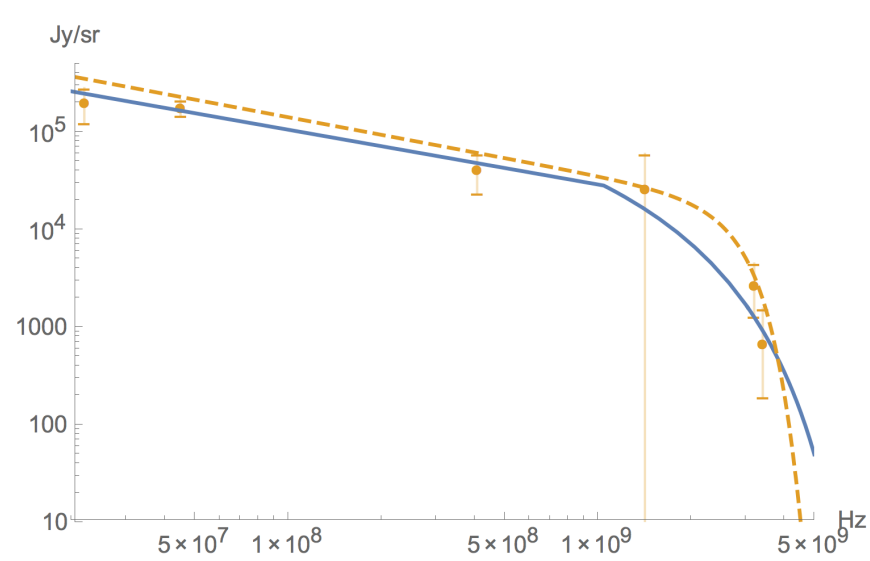

FIG. 3. The cosmic radio background due to fast radio transients (blue, solid) with cosmological rate based on the comoving volume, and the observed radio excess data points (orange) with error bars derived from the temperature measurements. The dashed orange line is from the continuous best fit function (power law in temperature) as reported by ARCADE 2. The excess may continue up to about $10 \mathrm{GHz}$, but significance at these high frequencies drops and the best fit from the fast transients has no power at these high frequencies. Note that at $1.4 \mathrm{GHz}$ the error bar is consistent with zero, where we've subtracted known radio sources as in [24].

for the simple model (the total luminosity $\mathcal{L} \times$ rate $R_{\mathrm{FRT}}$, the spectrum endpoints, and the spectral index), we find a $\chi^{2}$ per degree of freedom of about 4.03/4. The spectral index is 0.56 and the spectrum goes from $10 \mathrm{MHz}$ to $7.3 \mathrm{GHz}$ with a normalization of $4.6 \times 10^{35} \mathrm{~J} \mathrm{~Hz}^{-1}$. Note that the fitted spectrum cuts off at high frequencies before the final data points at 7.98 and $8.33 \mathrm{GHz}$ reported by ARCADE 2 to have an excess temperature, but the reported excess of these points are highly suppressed (of lower significance, and consistent with zero) compared to the other points. Plots of the fitted model and radio excess are shown in Figure 3.

For the cosmological fast transient rate modeled after the star formation rate, the results are similar: The fitted spectrum again has spectral index 0.56, normalization $4.2 \times 10^{36} \mathrm{~J} \mathrm{~Hz}^{-1}$, and an overall $\chi^{2}$ per degree

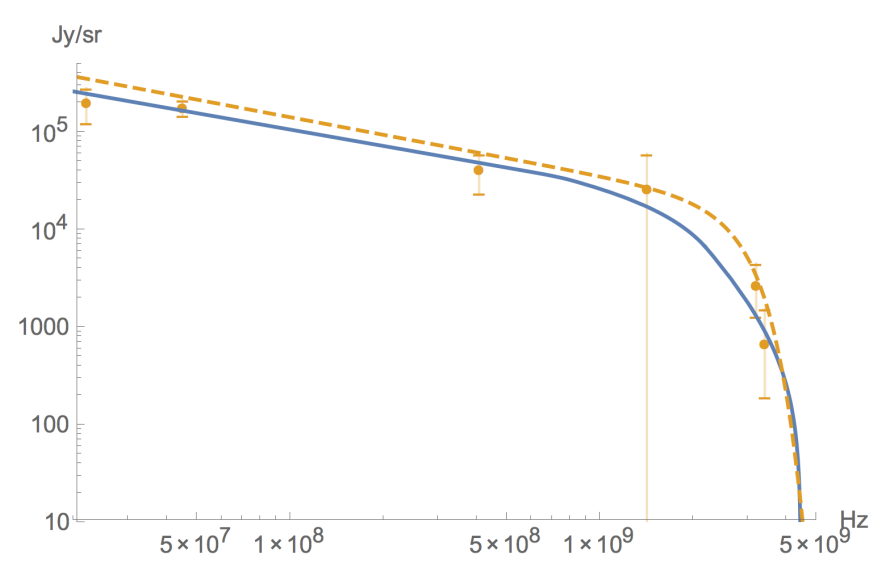

FIG. 4. The same as in Figure 3 but with the fast radio transient rate proportional to the star formation rate.

of freedom of 3.96/4. The fitted lower frequency of the spectrum is the same as the previous case, but the upper endpoint is $4.5 \mathrm{GHz}$. This model is plotted in Figure 4 . We note that the largest difference between this background model and the previous one is in the overall normalization of the spectrum, which needs to be about an order of magnitude larger in this case.

\section{DISCUSSION}

We turn now to interpreting the parameters of the best fit found above. Compared to the model spectral index of -1.4 for FRBs, the spectral index of the best fit is very flat in order to match the observed radio background. And, as we know from the FRB results, we need an overall factor of about 7 orders of magnitude larger to completely saturate the radio background excess. Alternatively, this factor needs to be at least 5 orders of magnitude larger than FRBs in order to be at the percent level of the radio excess.

This normalization is the combination of two main factors: the event rate, and overall energy. One could also subdivide this into further details, such as possible re- 
currence rate, luminosity dependence on redshift (i.e. a distribution of observed source energies), and so on.

While there have been searches for FRTs in the past (see the recent review in [19]), much of parameter space is unexplored and unconstrained. Searches are also usually done in narrow frequency bands, and are not sensitive to general combinations of parameters (e.g. minimum peak flux density versus event time). This makes it particularly difficult to draw general conclusions and constraints on what FRT parameters are currently allowed, though the flat fitted spectrum here may already be constrained. Due to the recent discovery and study of FRBs, this set of parameters (bright millisecond sources at $1.4 \mathrm{GHz}$ ) is better studied. From our analysis above, it looks rather unlikely that FRBs, or sources very similar to FRBs, play a role in the ERB.

On the other hand, there is still much more to be explored. Monochromatic sources will be very easy to miss without searching at exactly the right frequency, for instance. While a single such source could not explain the entirety of the ERB, perhaps multiple types of sources play some part. It is not difficult to find parameters for FRTs that avoid current constraints (which, we stress, are typically not very general), especially rare very bright sources (perhaps peaking at unexplored frequencies), or very dim sources. A comprehensive analysis of the available parameter space, and potential FRT models, is outside the scope of this current work, but is a promising future direction. With instruments such as the Square Kilometer Array aiming to search for radio transients, the coming years may yield many new discoveries.

\section{CONCLUSIONS}

In this work our primary aim has been to extend the analysis of sources which can contribute to a radio background to include FRTs. These have not been considered before in relation to the cosmic radio background. With large swaths of parameter space available, FRTs may play a role in the ARCADE 2 (and older experiments) excess. To that end we have used both an estimate based on a differential flux density and a detailed calculation for transient sources to calculate the contribution to a radio background.

This analysis has shown that based on what we currently know about $\mathrm{FRBs}$, they do not contribute at an appreciable level to the radio excess measurement. One could construct very different source models which could give a contribution to (or explanation of) the ERB, and such model building is one possible direction to studying fast transients and the radio background.

Overall, this work is meant to serve as a first step in a new direction for transient radio sources and the unknown cosmic radio background. There is ample opportunity here to propose fast radio transient models, search and hopefully discover their origin, and perhaps link them to the observed, yet unexplained, radio background.

\section{ACKNOWLEDGMENTS}

The authors were supported in part by the DOE (DESC0011981). TKW and TJW thank the Aspen Center for Physics, which is supported by NSF grant PHY-1066293, for hospitality while this work was in progress.
[1] D. J. Fixsen, A. Kogut, S. Levin, M. Limon, P. Lubin, P. Mirel, M. Seiffert, J. Singal, E. Wollack, T. Villela, and C. A. Wuensche, "Arcade 2 measurement of the absolute sky brightness at 3-90ghz," The Astrophysical Journal 734, 5 (2011).

[2] J. Singal, . Stawarz, A. Lawrence, and V. Petrosian, "Sources of the radio background considered," Monthly Notices of the Royal Astronomical Society 409, 11721182 (2010).

[3] M. Seiffert, D. J. Fixsen, A. Kogut, S. M. Levin, M. Limon, P. M. Lubin, P. Mirel, J. Singal, T. Villela, E. Wollack, and C. A. Wuensche, "Interpretation of the arcade 2 absolute sky brightness measurement," The Astrophysical Journal 734, 6 (2011).

[4] Nicolao Fornengo, Roberto A. Lineros, Marco Regis, and Marco Taoso, "The isotropic radio background revisited," Journal of Cosmology and Astroparticle Physics 2014, 008 (2014).

[5] T. Vernstrom, Douglas Scott, and J. V. Wall, "Contribution to the diffuse radio background from extragalactic radio sources," Monthly Notices of the Royal Astronomical Society 415, 3641-3648 (2011).
[6] Gilbert P. Holder, "The unusual smoothness of the extragalactic unresolved radio background," The Astrophysical Journal 780, 112 (2014).

[7] N. Fornengo, R. Lineros, M. Regis, and M. Taoso, "Possibility of a Dark Matter Interpretation for the Excess in Isotropic Radio Emission Reported by ARCADE," Phys.Rev.Lett. 107, 271302 (2011), arXiv:1108.0569 [hep-ph]; "Cosmological Radio Emission induced by WIMP Dark Matter," JCAP 1203, 033 (2012), arXiv:1112.4517 [astro-ph.CO]; Dan Hooper, Alexander V. Belikov, Tesla E. Jeltema, Tim Linden, Stefano Profumo, et al., "The Isotropic Radio Background and Annihilating Dark Matter," Phys.Rev. D86, 103003 (2012), arXiv:1203.3547 [astro-ph.CO]; Yupeng Yang, Guilin Yang, Xiaoyuan Huang, Xuelei Chen, Tan Lu, et al., "Contribution of ultracompact dark matter minihalos to the isotropic radio background," Phys.Rev. D87, 083519 (2013), arXiv:1206.3750 [astro-ph.HE]; Kyle Lawson and Ariel R. Zhitnitsky, "Isotropic Radio Background from Quark Nugget Dark Matter," Phys.Lett. B724, 17-21 (2013), arXiv:1210.2400 [astro-ph.CO]; James M. Cline and Aaron C. Vincent, "Cosmological 
origin of anomalous radio background," JCAP 1302, 011 (2013), arXiv:1210.2717 [astro-ph.CO].

[8] D. R. Lorimer, M. Bailes, M. A. McLaughlin, D. J. Narkevic, and F. Crawford, "A bright millisecond radio burst of extragalactic origin," Science 318, 777-780 (2007).

[9] E. F. Keane, B. W. Stappers, M. Kramer, and A. G. Lyne, "On the origin of a highly dispersed coherent radio burst," Monthly Notices of the Royal Astronomical Society: Letters 425, L71-L75 (2012).

[10] D. Thornton, B. Stappers, M. Bailes, B.R. Barsdell, S.D. Bates, et al., "A Population of Fast Radio Bursts at Cosmological Distances," Science 341, 53-56 (2013), arXiv:1307.1628 [astro-ph.HE].

[11] L.G. Spitler, J.M. Cordes, J.W.T. Hessels, D.R. Lorimer, M.A. McLaughlin, et al., "Fast Radio Burst Discovered in the Arecibo Pulsar ALFA Survey," Astrophys.J. 790, 101 (2014), arXiv:1404.2934 [astro-ph.HE].

[12] D. G. P. Thornton, The High Time Resolution Radio Sky, Ph.D. thesis, The University of Manchester (2013); Sarah Burke-Spolaor and Keith W. Bannister, "The Galactic Position Dependence of Fast Radio Bursts and the Discovery of FRB011025," Astrophys. J. 792, 19 (2014), arXiv:1407.0400 [astro-ph.HE]; E. Petroff, M. Bailes, E. D. Barr, B. R. Barsdell, N. D. R. Bhat, et al., "A realtime fast radio burst: polarization detection and multiwavelength follow-up," Mon. Not. Roy. Astron. Soc. 447, 246-255 (2015), arXiv:1412.0342 [astro-ph.HE]; V. Ravi, R. M. Shannon, and A. Jameson, "A fast radio burst in the direction of the Carina dwarf spheroidal galaxy," Astrophys. J. 799, L5 (2015), arXiv:1412.1599 [astroph.HE].

[13] S. R. Kulkarni, E. O. Ofek, J. D. Neill, Z. Zheng, and M. Juric, "Giant Sparks at Cosmological Distances?" Astrophys. J. 797, 70 (2014), arXiv:1402.4766 [astroph.HE]; D. Maoz, A. Loeb, Y. Shvartzvald, M. Sitek, M. Engel, et al., "Fast radio bursts: the observational case for a Galactic origin," (2015), arXiv:1507.01002 [astro-ph.SR].

[14] Tomonori Totani, "Cosmological fast radio bursts from binary neutron star mergers," Publications of the Astronomical Society of Japan 65 (2013), 10.1093/pasj/65.5.L12; Heino Falcke and Luciano Rezzolla, "Fast radio bursts: the last sign of supramassive neutron stars," Astron. Astrophys. 562, A137 (2014), arXiv:1307.1409 [astro-ph.HE].

[15] A. Iwazaki, "Axion stars and fast radio bursts," Phys. Rev. D91, 023008 (2015), arXiv:1410.4323 [hep-ph]; I. I.
Tkachev, "Fast Radio Bursts and Axion Miniclusters," JETP Lett. 101, 1-6 (2015), arXiv:1411.3900 [astroph.HE].

[16] V. M. Lipunov and M. V. Pruzhinskaya, "Scenario Machine: fast radio bursts, short gamma-ray burst, dark energy and Laser Interferometer Gravitational-wave Observatory silence," Mon. Not. Roy. Astron. Soc. 440, 1193 1199 (2014), arXiv:1312.3143 [astro-ph.HE].

[17] S. B. Popov and K. A. Postnov, "Millisecond extragalactic radio bursts as magnetar flares," (2013), arXiv:1307.4924 [astro-ph.HE]; Kazumi Kashiyama, Kunihito Ioka, and Peter Mszros, "Cosmological Fast Radio Bursts from Binary White Dwarf Mergers," Astrophys. J. 776, L39 (2013), arXiv:1307.7708 [astro-ph.HE]; Liam Connor, Jonathan Sievers, and Ue-Li Pen, "NonCosmological FRB's from Young Supernova Remnant Pulsars," (2015), arXiv:1505.05535 [astro-ph.HE]; J. I. Katz, "Inferences from the Distributions of Fast Radio Burst Pulse Widths, Dispersion Measures and Fluences," (2015), arXiv:1505.06220 [astro-ph.HE].

[18] A. Rane, D. R. Lorimer, S. D. Bates, N. McMann, M. A. McLaughlin, and K. Rajwade, "A search for rotating radio transients and fast radio bursts in the Parkes highlatitude pulsar survey," (2015), arXiv:1505.00834 [astroph.HE].

[19] Rob Fender, Adam Stewart, Jean-Pierre Macquart, Immacolata Donnarumma, Tara Murphy, et al., "Transient Astrophysics with the Square Kilometre Array," (2015), arXiv:1507.00729 [astro-ph.HE].

[20] Cecilia Lunardini, "Diffuse supernova neutrinos at underground laboratories," (2010), arXiv:1007.3252 [astroph.CO].

[21] D. R. Lorimer, A. Karastergiou, M. A. McLaughlin, and S. Johnston, "On the detectability of extragalactic fast radio transients," Monthly Notices of the Royal Astronomical Society: Letters 436, L5-L9 (2013).

[22] Andrew M. Hopkins and John F. Beacom, "On the normalisation of the cosmic star formation history," Astrophys. J. 651, 142-154 (2006), arXiv:astro-ph/0601463 [astro-ph].

[23] M. Gervasi, A. Tartari, M. Zannoni, G. Boella, and G. Sironi, "The contribution of the unresolved extragalactic radio sources to the brightness temperature of the sky," The Astrophysical Journal 682, 223 (2008).

[24] J. J. Condon, W. D. Cotton, E. B. Fomalont, K. I. Kellermann, N. Miller, R. A. Perley, D. Scott, T. Vernstrom, and J. V. Wall, "Resolving the radio source background: Deeper understanding through confusion," The Astrophysical Journal 758, 23 (2012). 